\title{
Design and implementation of participatory hygiene and sanitation transformation (PHAST) as a strategy to control soil-transmitted helminth infections in Luweero, Uganda
}

\author{
Dumba $\mathrm{R}^{1}$, Kaddu JB${ }^{2}$, *Wabwire-Mangen $\mathrm{F}^{3}$
}

1. Department of Biological Sciences, Kyambogo University, Uganda

2. Department of Zoology, Makerere University, Kampala, Uganda

3. School of Public Health, College of Health Sciences, Makerere University, Kampala, Uganda

\begin{abstract}
Background: The study is a continuation of a research carried out in Luweero district in Uganda ${ }^{1}$. It investigated whether PHAST was a suitable tool for reducing transmission of soil transmitted helminths. PHAST means Participatory Hygiene and Sanitation Transformation; a participatory approach that uses visual tools to stimulate the participation of people in promotion of improved hygiene and sanitation.

Objective: To assess the effect of PHAST on intestinal helminth transmission in children under five years.

Methods: Three phases namely; (1) Baseline survey (2) PHAST intervention (3) Follow up were conducted. During Phase 1 , the subjects' stool samples were examined for presence of helminthic ova and questionnaires administered. In Phase 2 , PHAST was conducted only in experimental villages. All subjects in the experimental and control villages were treated thrice with Albendazole. During Phase 3, all steps of Phase 1 were repeated.

Results: There was an overall reduction in the prevalence of children infected with helminths after PHAST intervention. Also, comparison of pre-intervention and post-intervention multivariate results indicates that the likelihood of children getting infected with helminths reduced in most of the experimented variables.

Conclusion: Health stakeholders should utilize PHAST approach to sensitize communities on the importance of hygiene to curb soil-transmitted helminth infections.

Key words: PHAST intervention, soil-transmitted helminth infections, Luweero district, Uganda.

African Health Sciences 2013; 13(2): 512 - 517 http:/ /dx.doi.org/10.4314/ahs.v13i2.44
\end{abstract}

\section{Introduction}

Intestinal helminth infections have been reported in many parts of Uganda including Luweero district $t^{1-4}$. The district has had inadequate investigations on the problem, yet it has had the infections for quite a long time ${ }^{3}$; it was therefore necessary to investigate a way of controlling its transmission. Thus, this study was conducted to assess the effect of PHAST intervention on intestinal helminth infections in children less than 5 years. PHAST is a participatory approach, which was developed to encourage people to analyze their own situation and identify key problems, decide what things need to be improved, plan how they are going to do it and then act. For many years, conventional messages on hygiene and sanitation had been known

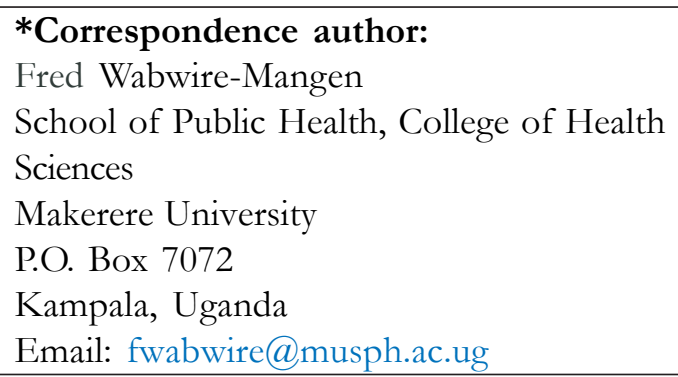

and largely understood by people. However, these messages had not translated into significant improvement in good hygiene practices.

In 1993, WHO and the Regional Water and Sanitation Group for East and Southern Africa (RWSG-ESA) initiated the PHAST strategy to address this concern ${ }^{5}$. Among the PHAST tools that were developed, 4 were purposely selected as most appropriate for the current study to cover 5 of the essential steps to community planning namely; Sanitation ladder, Three-Pile Sorting, Faecal-Oral disease Transmission Routes and Barriers (FTRB), and Tippy Tap ${ }^{6}$. Sanitation ladder (set of pictures showing various methods of excreta disposal) was selected for problem identification.

Participants arranged pictures in form of ladder, from the worst practice/latrine to the best; identifying their own situation and looking at advantages of moving up the ladder. Three-Pile Sorting was for analyzing problems and selecting options; participants sorted out 30 pictures of hygiene/sanitation related situations depending on 
whether they considered them "good", "bad" or "in-between" giving reasons in each case. FTRB was for planning for solutions; participants organized a set of pictures basing on what they knew about faecal-oral transmission routes and then worked out how to block the routes using common barrier pictures. Tippy tap was for planning for new facilities and behaviour change; this was a hand washing facility demonstrated to participants; they were encouraged to make and use it in their homes. Monitoring was done after every training session and family members assessed their progress using pictorial monitoring forms.

Despite the fact that PHAST had been employed in some programmes such as RUWASA, effects of the use of participatory methods had not been systematically monitored or documented. Recorded effects were merely anecdotal and it lacked baseline surveys to prove its effectiveness $s^{5,7}$.

This study therefore tested the approach as no studies had been carried out to assess its impact.

\section{Methods}

The study was a randomized community intervention trial with pre- and post-intervention phases. The study was implemented in 3 phases. Phase 1 was a baseline cross-sectional descriptive survey that investigated the prevailing helminth status described in already published paper ${ }^{1}$. Two sub-counties, selected by simple random sampling, consisted of 4 parishes from which 19 study villages were studied. Stool samples from 727 eligible children were examined for presence of different types of helminth ova using Kato-Katz ${ }^{8}$ technique. Semi-structured questionnaires were also administered to parents/ guardians and inspection of households conducted to assess their hygiene status.
During Phase 2, the four parishes were randomly assigned 10 experimental and 9 control villages with 357 and 370 children respectively. PHAST health education was carried out thrice among the experimental group (parents/guardians) only. After each training session, the respondents' households were visited to reinforce what had been discussed during the training. At each visit, household members freely discussed and identified their household sanitation and hygiene status they had attained plus the ones they were aiming at using pictorial monitoring forms. In addition, all the children were treated with a single oral dose of Albendazole depending on age once every 3 months; those below 2 years were given one 200-mg tablet whereas those between 2 and 5 years two 200-mg tablets. It was a directly observed therapy.

During Phase 3, all steps and procedures in Phase 1 were repeated. The results were analyzed using univariate and bivariate analyses. Chi-square test was used and the level of significance set to $95 \%$ level. The relationship between the variables was established as statistically significant when found to be equal to or less than $0.05^{\circ}$. The odds ratios (OR) of the children were determined using the odds ratio statistic in a $2 \times 2$ analysis. Multivariate analysis was further used to investigate how helminth infection was related to more than one variable at a time while controlling for confounders. A binary logistic regression model was used to obtain the adjusted OR.

\section{Results}

Table 1 indicates that the prevalence rate of children infected with helminth ova which was $27.6 \%$ (201/ 727) at baseline reduced to $16.5 \%$ after PHAST intervention.

\section{Table 1: Comparison of Phases 1 and 3 helminth infections}

\begin{tabular}{|c|c|c|c|c|}
\hline \multirow[t]{2}{*}{ Type of worm } & \multicolumn{2}{|c|}{$\begin{array}{l}\text { No. of cases before intervention } \\
\text { - Phase } 1 \quad(\mathrm{n}=727)\end{array}$} & \multicolumn{2}{|c|}{$\begin{array}{l}\text { No. of cases after interventior } \\
\text { - Phase } 3(\mathrm{n}=558)\end{array}$} \\
\hline & No. of infections & $(\%)$ & No. of infections ${ }^{\mathrm{D}}$ & $(\%)$ \\
\hline Ancylostoma duodenale / Necator americans & 165 & $(82.1)$ & 64 & $(71.1)$ \\
\hline Ascaris lumbricoides & 38 & $(18.9)$ & 10 & $(11.1)$ \\
\hline Trichuris trichiura & 14 & $(7.0)$ & 22 & $(24.4)$ \\
\hline Hymenolepis nana & 1 & $(1.0)$ & 0 & 0 \\
\hline Enterobius vermicularis & 2 & $(0.5)$ & 5 & $(5.6)$ \\
\hline Total & 220 & 27.6 & 101 & 16.5 \\
\hline
\end{tabular}

P - Phase 3 percentages are based on a total of 92 infected cases, 101 includes double infections 
There was a high drop rate basically due to migration from study area; hence the difference in the two study populations during Phase $1 \& 3$ (727 and 558 respectively). Phase 3 data contains both experimental and control groups.

Comparison of Phase 1 and 3 pre-

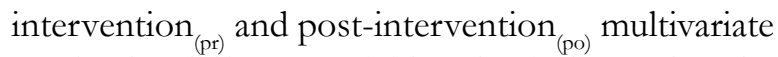
results for different variables also indicate that the odds of children getting infected with helminth ova reduced after PHAST intervention in all the variables except three; these either had an increase or a constant Odds Ratio (tables $2 \mathrm{a}$ and $2 \mathrm{~b}$ ).

If OR after PHAST is lower than OR before PHAST, there was a reduction in helminth infections. The variables that indicated the most significant reduction were; condition of latrines, respondents' hand washing after handling children's faeces and keeping of pigs. These variables were significantly associated with helminth infection before the intervention but were not associated after the intervention. For instance, there was nearly a threefold reduction in OR noted among helminth-infected children who were living in homes with poorly maintained latrines compared to those in homes with fairly maintained latrines $\left(\mathrm{OR}_{\mathrm{pr}}=1.90 ; 95 \% \mathrm{CI}=\right.$ $\left.1.17-3.10 \mathrm{Vs} \mathrm{OR}_{\mathrm{po}}=0.74 ; 95 \% \mathrm{CI}=0.30-1.80\right)$. Furthermore, of the children infected with helminths, the likelihood of those who lived in homes with pigs getting infected also decreased by a half after the intervention compared to those in homes without pigs, $\left(\mathrm{OR}_{\mathrm{pr}}=1.73 ; 95 \% \mathrm{CI}=1.17-2.58 \mathrm{Vs} \mathrm{OR}_{\mathrm{po}}=\right.$ $0.82 ; 95 \% \mathrm{CI}=0.48-1.39)$.

Table 3 indicates that generally, there was a statistically significant decline in prevalence in the control group (chi square $=16.90, \mathrm{p}=0.00)$.

Table 2a: Comparison of pre-intervention and post-intervention multivariate results

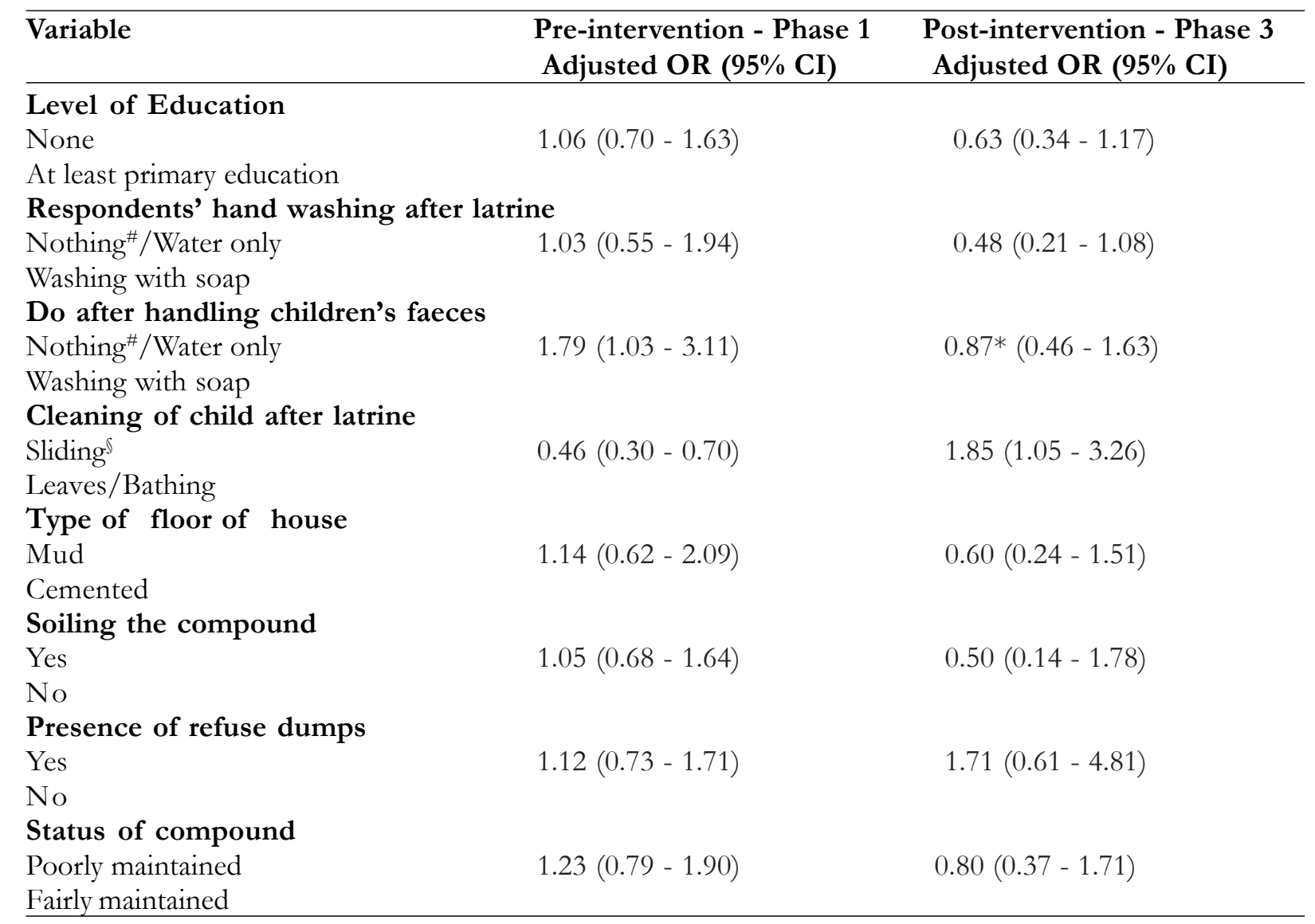

OR - Odds ratio CI - Cornfield 95\% confidence limits for OR \# - Respondents did not wash hands * - Most significant OR reductions after PHAST intervention $\checkmark$ - Children pushing against the ground as a way of cleaning themselves after visiting 
Table 2b: Comparison of pre-intervention and post-intervention multivariate results

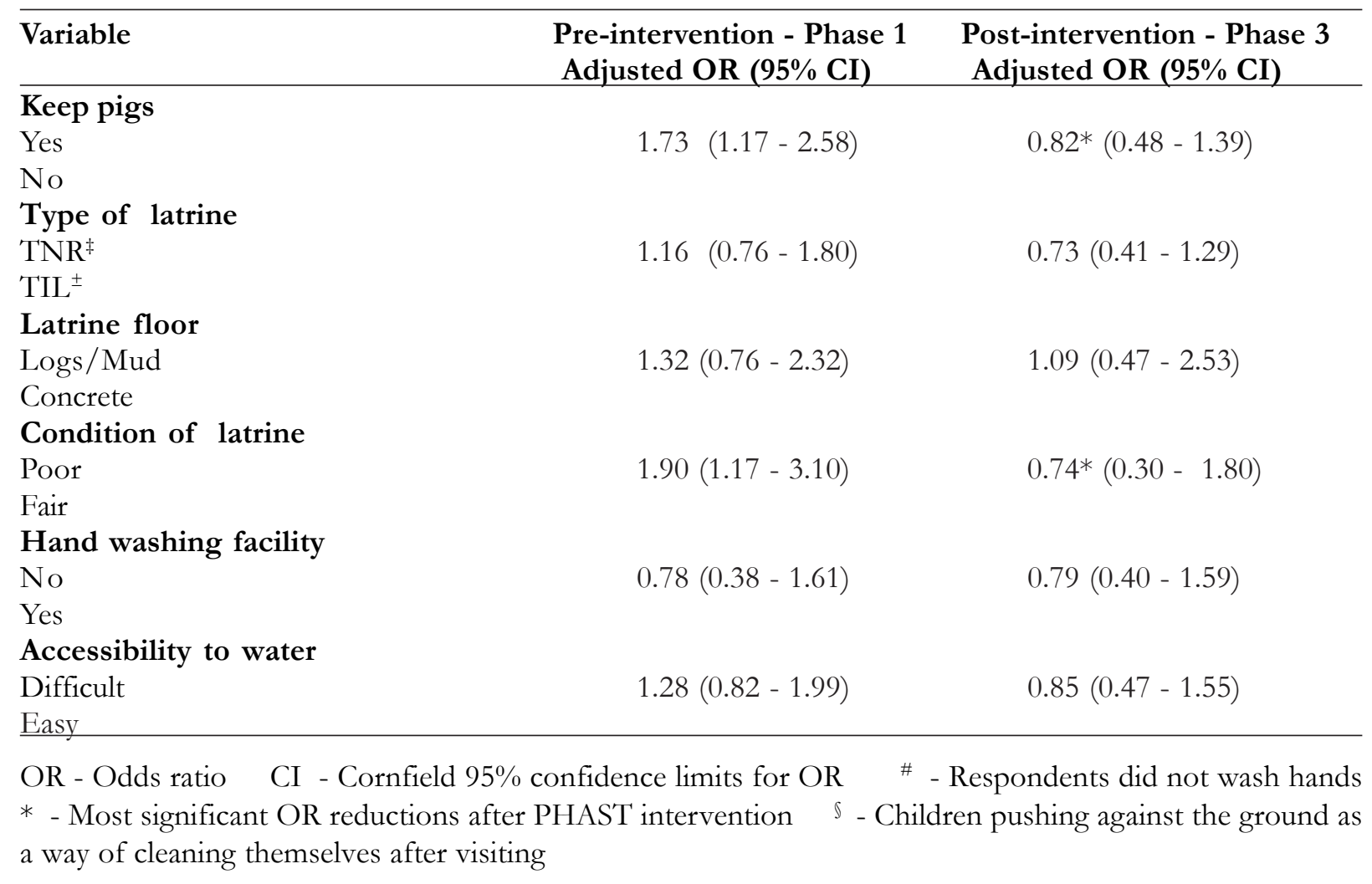

Table 3: Comparison of prevalence of helminth infections of the study groups for Phase $1^{¥}$ and Phase $3^{\odot}$

\begin{tabular}{|c|c|c|c|c|c|c|c|}
\hline \multirow[t]{2}{*}{ Study groups } & \multicolumn{2}{|c|}{ Prevalence $(\%)$} & \multicolumn{2}{|c|}{ Prevalence $(\%)$} & \multicolumn{2}{|c|}{ Percent Decline } & \multirow[b]{2}{*}{$\begin{array}{l}\text { Chi-Square } \\
\text { (P-value) }\end{array}$} \\
\hline & Per Parish & $\begin{array}{l}\text { Per study } \\
\text { Arm }\end{array}$ & $\begin{array}{l}\text { Per } \\
\text { Parish }\end{array}$ & $\begin{array}{l}\text { Per study } \\
\text { Arm }\end{array}$ & Per Parish & $\begin{array}{l}\text { Per study } \\
\text { Arm }\end{array}$ & \\
\hline \multirow[t]{2}{*}{ Control } & $(24.6)$ & 29.7 & $(10.2)$ & 15.9 & 14.4 & 14.5 & $\begin{array}{l}16.90 \\
(0.00)\end{array}$ \\
\hline & $(34.2)$ & & $(20.7)$ & & 13.5 & & \\
\hline \multirow[t]{2}{*}{ Experimental } & $\begin{array}{l}(12.3) \\
(38.8)\end{array}$ & 23.5 & $\begin{array}{l}(9.9) \\
(23.8)\end{array}$ & 17.1 & $\begin{array}{l}2.4 \\
15.0\end{array}$ & 8.5 & $\begin{array}{l}6.41 \\
(0.01)\end{array}$ \\
\hline & & 27.6 & & 16.5 & & 11.1 & $\begin{array}{l}1.83 \\
0.18\end{array}$ \\
\hline
\end{tabular}

${ }^{¥}$ Phases 1 - Pre-intervention $\quad{ }^{\circ}$ Phases 3 - Post-intervention $\quad \cdot$ Mantel - Haenszel’s Chi-square used

There was also a statistically significant decline in the prevalence in experimental group (Chi-square $=6.41$, $\mathrm{p}=0.01)$. A difference in the decline in prevalence for the control and experimental groups was noted but not statistically significant (The control group declined 6 percent points more than the experimental group $($ Chi-square $=1.83, \mathrm{p}>0.05)$.

\section{Discussion}

Effect of PHAST intervention on helminthic infections

The overall prevalence rate of helminth infections reduced after the intervention in Phase 3. The likelihood of children getting infected with helminths also reduced in almost all the variables. The observed reduction in these aspects is likely to be attributed to PHAST intervention. As stipulated by Simpson-Herbert et al., 19976, tools and techniques that were used during PHAST participatory health education in the experimental group could have stimulated participation even among participants who did not know how to read. By taking them through the various steps and activities, it was possible to help them understand that poor hygiene and sanitation behaviours and practices are the principal causes of many 
preventable diseases, such as helminthiasis. Whereas conventional health education, which is normally delivered by didactic teaching, is aimed at reducing transmission and re-infection by encouraging healthy behaviour, it is not normally presented as a user friendly package involving participants. UNDP recommends that participatory methods create a non-threatening environment in which all participants regardless of class, age and sex can express their views freely ${ }^{10}$. This freedom was observed during PHAST sessions where almost every participant was able to identify their own problems and plan for solutions in a relaxed atmosphere. Evidently, PHAST approach was an eye opener to the respondents as noted by a reduction in odds ratio after the intervention in most aspects. Reduction, for instance, was noted in the risk of children acquiring infections through respondents' hand washing with soap after visiting the latrine and after handling children's faeces.

Reduction in the likelihood of children getting infected with worms in homes with mud floors indicates that, despite the fact that floors remained uncemented, participants cleaned them better after PHAST exposure. Decrease in the risk of children acquiring infections for those living in homes with soiled compounds could be attributed to respondents' becoming aware that indiscriminate dumping of faeces was harmful and therefore ensured that it was properly disposed of. The improvement in the type of latrine, latrine floor and condition was linked with the newly constructed or improved latrines observed during Phase 2 monitoring. This in turn resulted in the reduction of risk of children acquiring worm infections. The decline in odds of children acquiring worm infection in homes keeping pigs could be attributed to respondents who could have restricted the roaming of pigs after the intervention, thus reducing on indiscriminate defaecation. According to $\mathrm{Scott}^{11}$, the association with pig ownership is intriguing giving the continuing interest in zoonotic potential of transmission of Ascaris from pigs to humans. The results of respondents who had difficulties in accessing water show that, despite the problem, they could have started using it sparingly for improving hygiene practices.

However, an increase in the risk of children acquiring worm infections for those who cleaned their anal region by pushing themselves against the ground (sliding) after visiting the latrine, could be attributed to the fact that it was not easy for the parents/guardians to teach their kids the proper way of cleaning themselves as they were either busy with domestic chores or away from home whenever they defaecated. The fact that the risk of children getting infected for those living in homes with refuse dumps increased whereas that of children in homes without hand washing facilities (tippy taps) remained the same after intervention could imply that more home visits were still required to sensitize respondents to clean up compounds and to produce more of such facilities.

In view of the findings discussed above that there were significant changes in hygiene related behaviour after the intervention, it is possible that within the short run, chemotherapy has a greater effect on worm prevalence generally than the PHAST intervention as indicated in Table 3. The increase in hygiene related behaviour in various variables, however, suggests that PHAST intervention would have a greater effect on worm prevalence but in a long run. In this respect, it should be noted that least decline was recorded in Kasala parish (2.4\%) where PHAST sessions were least attended and highest decline in Kisimula (15\%) where there was good attendance yet the parish had had highest prevalence $(38.8 \%)$ prior intervention.

\section{Conclusion}

It is possible that higher reductions would have been registered if PHAST activities were conducted for a longer period. Switzerland et al., 2010 ${ }^{12}$ reports that PHAST is a process that takes time. The study findings, therefore, raise questions on how long and how frequently PHAST has to be implemented in order to be fully effective. This requires further research. According to Loevinsohn ${ }^{13}$, successful health education depends on using a few messages, of proven benefit, repeatedly, and in many fora.

\section{References}

1. Dumba R, Kaddu JB, Wabwire-Mangen F. Intestinal helminths in Luweero district. African Health Sciences 2008; 8 (2): 90-96.

2. Lin CJ, Katongole-Mbidde E, Byekwaso T, Orem J, Rabkin CS, Mbulaiteye SM. Intestinal Parasites in Kaposi Sarcoma Patients in Uganda: Indication of Shared Risk Factors or Etiologic Association. Am. J. Trop. Med. Hyg., 2008; 78 (3): 409-412.

3. HMIS, Health Management Information System annual reports from Ministry of Health, Uganda, $1997-2005$. 
4. Mwebesa KT. Factors influencing the prevalence of intestinal helminths among pupils in Primary Schools in Tororo District, Uganda. DPH dissertation, Makerere University, Kampala, Uganda, 2001.

5. Obel-Lawson L. (Ed.). Participatory Hygiene and Sanitation in East and Southern Africa Prospective Review of Participatory Hygiene and Sanitation Transformation (PHAST). UNDP World Bank Water and Sanitation Program and WHO, 1998; 1-7.

6. Simpson-Herbert M, Sawyer R and Clarke L. The PHAST Initiative - Participatory Hygiene and Sanitation Transformation, a new approach to working with Communities. WHO Geneva and UNDP - World Bank. Water and Sanitation Program, 1997; 1-22.

7. UNDP. Participatory Hygiene and Sanitation in East and Southern Africa. Prospective Review of PHAST. UNDP - World Bank and Sanitation Program and WHO, 1998; 1-7.

8. Katz NA, Chaves A, Pellegrino JA. Simple device for quantitative stool thick smears technique in Schistosmiasis mansoni. Revista do
Instituto de Medicina Tropical de Sao Paul, 1972; 14: 397-400.

9. Morton RF, Hebel JR, McCarter JR. A study guide to Epidemiology and Biostatistics ( $5^{\text {th }} \mathrm{Ed}$.). Aspen Publishers, Incl. Maryland, 2001.

10. UNDP. Participatory Hygiene and Sanitation: The Future of PHAST Prospective Review. Report of the Consultative Meeting. UNDP - World Bank Water and Sanitation Program, WHO, UNICEF and RWSG-ESA, 1998; 4.

11. Scott ME. "Ascaris lumbricoides: A review of its epidemiology and relationship to other infections." Annales Nestle (Engl) 2008; 66: 7-22

12. Switzerland C, Lowe E, Omosa D. Participatory Hygiene and Sanitation Transformation Report in PHAST Training Workshop held in Nairobi, Kenya. UNICEF Somalia WASH Cluster 2010 Jan 12 - 20.

13. Loevinsohn BP. Health education Interventions in Developing Countries: A Methodological Review of Published Articles. International J. of Epidemiology, 1990; 19 (4): 788-792. 